\title{
Far-IR Measurements at Cerro Toco, Chile: FIRST, REFIR, and AERI
}

\author{
Richard P. Cageao*, J. Ashley Alford, David G. Johnson, David P. Kratz, \\ and Martin G. Mlynczak \\ NASA Langley Research Center, Hampton, VA
}

\begin{abstract}
In mid-2009, the Radiative Heating in the Underexplored Bands Campaign II (RHUBC-II) was conducted from Cerro Toco, Chile, a high, dry, remote mountain plateau, $23^{\circ} \mathrm{S}, 67.8^{\circ} \mathrm{W}$ at $5.4 \mathrm{~km}$, in the Atacama Desert of Northern Chile. From this site, dominant IR water vapor absorption bands and continuum, saturated when viewed from the surface at lower altitudes, or in less dry locales, were investigated in detail, elucidating IR absorption and emission in the atmosphere. Three FTIR instruments were at the site, the Far-Infrared Spectroscopy of the Troposphere (FIRST), the Radiation Explorer in the Far Infrared (REFIR), and the Atmospheric Emitted Radiance Interferometer (AERI). In a side-by-side comparison, these measured atmospheric downwelling radiation, with overlapping spectral coverage from 5 to100um (2000 to $100 \mathrm{~cm}^{-1}$ ), and instrument spectral resolutions from 0.5 to $0.64 \mathrm{~cm}^{-1}$, unapodized. In addition to the FTIR and other ground-based IR and microwave instrumentation, pressure/temperature/relative humidity measuring sondes, for atmospheric profiles to $18 \mathrm{~km}$, were launched from the site several times a day. The derived water vapor profiles, determined at times matching the FTIR measurement times, were used to model atmospheric radiative transfer. Comparison of instrument data, all at the same spectral resolution, and model calculations, are presented along with a technique for determining adjustments to line-by-line calculation continuum models. This was a major objective of the campaign
\end{abstract}

\section{Introduction}

\section{RHUBC-II}

The Radiative Heating in Underexplored Bands Campaign (RHUBC) is a multi-phase campaign designed to observe downwelling atmospheric infrared radiance. RHUBC field locations and observing times are chosen for their extreme dryness, providing primary absorber, spectroscopic $\mathrm{H}_{2} \mathrm{O}$ vibrational and rotational band information, not available at sea-level or in wetter environments in the near to far-IR. This spectral information provided a stringent test of line-by-line radiative transfer models. RHUBC- I occurred at the Atmospheric Radiation Measurement Program's (ARM) North Slope of Alaska Climate Research Facility (NSA) from February 22 to March 14, 2007, located outside Barrow, Alaska. The RHUBC-II Cerro Toco site was on a high $(5.4 \mathrm{~km})$, relatively remote mountain plateau in the Atacama Desert of Northern Chile $\left(23^{\circ} \mathrm{S}, 67.8^{\circ} \mathrm{W}\right)$, and was chosen because of the nearby Atacama Large Millimeter Array (ALMA) facility infrastructure, and because winter precipitable water vapor (PWV) values can dip to $0.04 \mathrm{~cm}$. The objectives for the August through October 2009 RHUC-II campaign, in

* Corresponding Author: Richard Cageao, Richard.P.Cageao@nasa.gov 
addition to an instrument data intercomparison, included refinement of both the water vapor continuum model and water vapor line parameters in the far-IR.

Three interferometric spectrometers deployed to the site, the Far-Infrared Spectroscopy of the Troposphere (FIRST), ${ }^{1}$ the Atmospheric Emitted Radiance Interferometer (AERI), ${ }^{2}$ and the Radiation Explorer in the Far-InfraRed (REFIR),3 together measured nearly the entire spectrum of earth's atmospheric downwelling radiation. In addition, PWV at the site was retrieved from the $183.31 \mathrm{GHz}$ Gband Vapor Radiometer (GVR) (uncertainty 2\%) and pressure, temperature, and humidity Vaisala RS92 altitude profiling sondes were launched nearly hourly to correspond with spectroscopic instrument observations. Water vapor data was used as input to atmospheric radiative transfer models to test measured versus modeled radiance. This infrared radiative closure experiment concept, using radiance measurements and line-by-line (LBL) radiative transfer model calculations, is outlined by Clough et al. ${ }^{4}$ and has been successfully used validate and improve continuum absorption models and spectral line parameters used in line-by-line models. Preliminary data analysis is presented (measurement - model calculation) comparing radiometric measurements themselves as well as the specification of water vapor absorption in a LBL model. Far-IR water vapor continuum model updates implied by FIRST data analysis are discussed.

\section{Scientific Background}

The observed downwelling atmospheric radiation is a significant component of the earth's atmospheric greenhouse warming, dependent on received solar visible and ultraviolet flux as well as the re-emitted earth's surface infrared and the atmospheric absorbers trapping this radiation, and their distribution in the troposphere. Because of the availability of detectors and techniques, and interest in atmospheric soundings for temperature determination and weather prediction, the downwelling and upwelling infrared radiation from the atmosphere are relatively well observed and modeled, at least in a non-absolute sense, below a wavelength of $15 \mu \mathrm{m}\left(667 \mathrm{~cm}^{-1}\right)$. However, at longer wavelengths (the far-IR), where the Planck function peaks at approximately $20 \mu \mathrm{m}\left(500 \mathrm{~cm}^{-1}\right)$ given the average $255 \mathrm{~K}$ atmospheric temperature, there have been very few measurements. Various estimates ${ }^{5-7}$ put the radiance in this portion of the spectrum at $50 \%$ of the total trapped by the atmosphere and therefore resulting in atmospheric heating. Without comparative data, atmospheric line-by-line radiative transfer models, dependent on relatively short path laboratory-derived line positions and line-shapes, cannot be shown to deliver validated results. These models are a source of predictions of atmospheric heating, and therefore climate change. In particular, then, these models need observations to validate the atmospheric radiative transfer calculations in the farIR.

The far-infrared has rarely been directly observed from space platforms or the surface. The Clouds and Earth's Radiant Energy System (CERES) ${ }^{8}$ instruments measure the total spectrally integrated emission shortward of $100 \mu \mathrm{m}$ and the integrated emission in the atmospheric window between 8 and $12 \mu \mathrm{m}$, with no further spectral distinction. The far-infrared (to $25 \mu \mathrm{m}$ ) was last directly observed from space with spectral discrimination by the IRIS instruments on the Nimbus series of satellites in 1969 and 1970 9,10 and by instruments on the Russian Meteor series of spacecraft in the mid-1970's. ${ }^{11}$ This paper 
documents an intercomparision of instruments at the mobile DOE/ARM Cerro Toco site in the Atacama desert, measuring downward infrared radiance in the mid- and far-IR, and focusing on overlapping spectral regions plus radiative modeling improvements that can be obtained from this data, most notably in the newly observed far-IR.

\section{Instrument Descriptions}

In the operations at the Cerro Toco site, the included preliminarily analyzed data came from three infrared FTS instruments:

\begin{tabular}{|c|c|c|c|}
\hline Characteristic & FIRST & AERI & REFIR \\
\hline Instrument Type & 2-port Plane-Mirror FTS & 4-port Corner Cube FTS & 4-port Corner Cube FTS \\
\hline Optical Throughput & $0.47 \mathrm{~cm}^{2}$ ster & $0.063 \mathrm{~cm}^{2}$ ster & $0.01 \mathrm{~cm}^{2}$ ster \\
\hline Spectral Range & $80-800 \mathrm{~cm}^{-1}$ & $500-2000 \mathrm{~cm}^{-1}$ & $210-1100 \mathrm{~cm}^{-1}$ \\
\hline Resolution (unapodized) & $0.643 \mathrm{~cm}^{-1}$ & $0.48 \mathrm{~cm}^{-1}$ & $0.5 \mathrm{~cm}^{-1}$ \\
\hline Detectors & $10 \mathrm{Si}$ bolometers & $\mathrm{HgCdTe} / \mathrm{InSb}$ & DLATGS pyroelectric \\
\hline Beamsplitter & Ge on polypropelene & $\mathrm{KBr}$ & Polyethelene (mylar) \\
\hline $\begin{array}{c}\text { Scan Time (double- } \\
\text { sided interferogram) }\end{array}$ & $8.5 \mathrm{sec}$ & $1 \mathrm{sec}$ & 64 sec \\
\hline Sky Integration & $6 \mathrm{~min}$. & $3.3 \mathrm{~min}$ & 6 min. \\
\hline Calib. Blackbodies & 46 and $12 \mathrm{C}$ & 60 and $-10 \mathrm{C}$ & 80 and $13 \mathrm{C}$ \\
\hline Period between Calibs. & $35 \mathrm{~min}$. & $8 \mathrm{~min}$. & $11 \mathrm{~min}$. \\
\hline Lab Calib. Uncertainty & $1 \mathrm{~K}$ & $0.1 \mathrm{~K}$ & $0.1 \mathrm{~K}$ \\
\hline
\end{tabular}

Table 1: Fourier Transform Spectrometers for mid- to far-IR measurements at Cerro Tocco site

Calibrations for each instrument included detector non-linear frequency and phase response, individual interferogram phase corrections for frequency dispersion, sampling, and other linear and non-linear phase offsets, two temperature blackbody calibration for determination of responsivity and offset and extrapolation to cooler atmospheric temperatures, ${ }^{12}$ and summing and averaging over the sky integration period. Additionally, FIRST corrects for off-axis frequency shits and vignetting in the scene select mirror and sums multiple detectors for increased precision, AERI corrects for field of view self-apodization instrument line shape (ILS) effects and wavelength scale errors, ${ }^{13}$ and REFIR resamples its interferograms from equal-time spaced points to equal position spaced points, corrects its ILS, and estimates the instrument noise equivalent spectral radiance (NESR) and overall calibration errors. ${ }^{14}$ In a radiometric sense, the uncertainties quoted above are for stable-source, laboratory radiometric measurements and may be differ in an environment of uncontrolled variables.

In addition, Vaisala RS92 pressure, temperature, and relative humidity sondes were launched at 1 hour intervals from the Cerro Toco site during infrared data acquisition. Data, to an approximate $17 \mathrm{~km}$ termination altitude after the $\sim 45 \mathrm{~min}$ ascent from $5.4 \mathrm{~km}$, for each sonde, was recorded via telemetered signal to a Cerro Toco site ground station computer. Relative humidity values measured by the sondes 
were scaled to GVR measured precipitable water vapor columns and also adjusted to remove sonde biases. Resultant sonde measurements have an estimated combined relative humidity uncertainty of $\pm 5.5 \% .^{15}$

Adjusted sonde RH values, plus temperature and pressure measurements, were later used as inputs to the NASA Langley Monochromatic Radiative Transfer Algorithm (MRTA) ${ }^{5}$ LBL calculation code. This LBL code has been intercompared with six other common radiative transfer calculation codes in the farIR $\left(100-650 \mathrm{~cm}^{-1}\right)$ with integrated radiance differences for the conditions at Cerro Toco that not exceed $0.3 \%{ }^{16}$. MRTA applies the 2008 HITRAN database for atmospheric line positions, strengths, and shapes, ${ }^{17}$ and adds a continuum absorption model (MT_CKD 2.1) for far wing absorptions developed for the LBLRTM radiative transfer code. ${ }^{18,19}$ Included as inputs to the MRTA calculation are recently measured (2008) atmospheric abundances of several key IR absorbers $\left(\mathrm{CO}_{2}, \mathrm{CH}_{4}, \mathrm{~N}_{2} \mathrm{O}, \mathrm{CO}, \mathrm{CFC}-11\right.$, $\mathrm{CFC}-12$, CFC-22, $\mathrm{CCl}_{4}, \mathrm{CF}_{4}$, and $\mathrm{SF}_{6}$ ) and an $\mathrm{O}_{3}$ profile typical of mid-latitude winter conditions.

\section{Data Comparisons}

\subsection{FIRST Data and AERI Data}

Figure 1

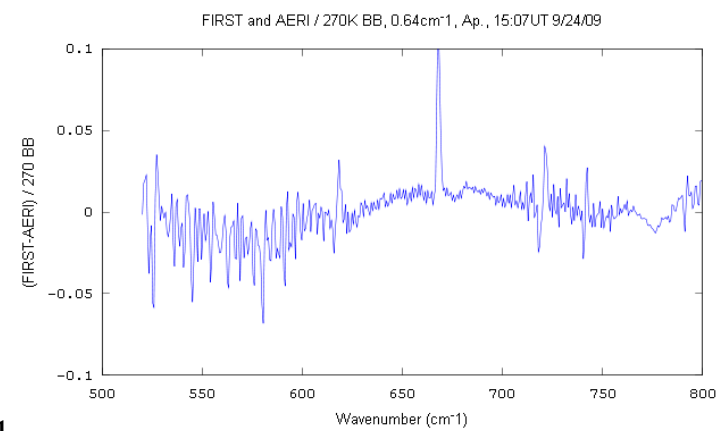

FIRST and AERI data, here shown at a nearly coincident time, 15:07UT on Sept. 24, 2009, are differenced and ratioed to a $270 \mathrm{~K}$ Planck function blackbody (the temperature that best fits the observed envelope of the data). Peak to peak differences do not exceed 7\%, except in the center of the $\mathrm{CO}_{2}$ band $\left(667 \mathrm{~cm}^{-1}\right)$ where the absorption saturates just outside the instrument window. The large peak to peak differences most commonly occur on the shoulders, in other words in the wings, of the absorption features in the data overlap region of $500-800 \mathrm{~cm}^{-1}$. Although in this and the following data and model comparison, care was taken in the calculation of spectral resolution (degrading AERI data to $0.643 \mathrm{~cm}^{-1}$ ) and in applying the same van Hann apodizing functions, some of these could be caused by slight registration errors in the wavenumber scale of the instrument data. This was recognized in the AERI data and recent corrections to the preliminary data wavenumber scale have been posted. Outside these rapidly varying absorption edges, differences are on the order of $2-3 \%$ (in radiance, equivalent to an approximately $2 \mathrm{~K}$ blackbody difference).

\subsection{FIRST Data and REFIR Data}




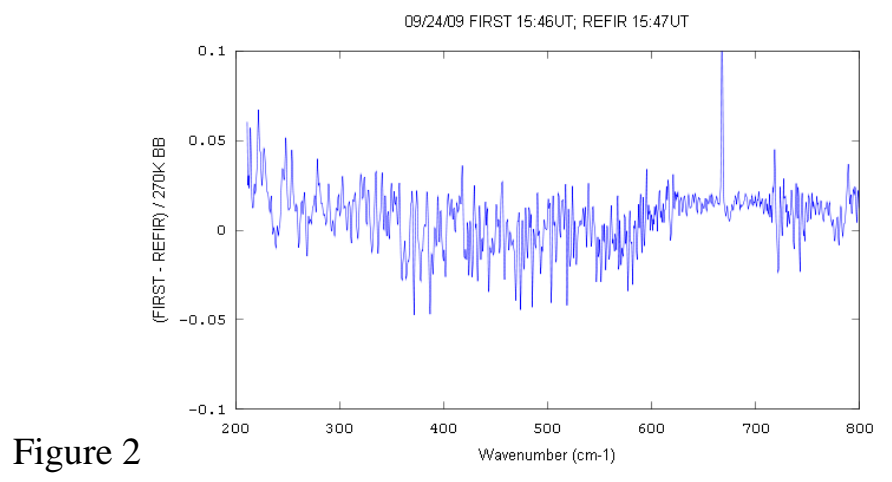

FIRST and REFIR data, here shown at a nearly coincident time, 15:47UT on Sept. 24, 2009, are differenced and ratioed to a 270K Planck function blackbody. REFIR data resolution was reduced to FIRST resolution $(0.643 \mathrm{~cm}-1)$ and a von Hann apodizing function was applied to both before differencing. As with the AERI data peak to peak differences do not exceed 7\%. After the issuance of the preliminary REFIR data set analysis revealed the need for an instrument line shape correction yet to be applied to the data set. This potential atmospheric line shape distortion may explain why the differences most commonly occur on the shoulders, or in the wings of the absorption features in the data overlap region of $210-800 \mathrm{~cm}^{-1}$. Outside the absorption feature wings differences again are on the order of $2-3 \%$ (in radiance, equivalent to an approximately $2 \mathrm{~K}$ blackbody difference).

\section{Model Comparison}

\subsection{The MRTA Model}

With inputs described above, and from the sondes flown at coincident times with the ground-based instrument data acquisition, forward modeled atmospheric downwelling radiances were calculated. In general, the atmosphere above the site was not static. Water vapor varied during the sonde ascent, even on the driest days $(0.04 \mathrm{~cm}$ PWV) and this is reflected in the acquired data radiances over the sky integration period ( $6 \mathrm{~min}$. for FIRST and REFIR, $3.3 \mathrm{~min}$. for AERI) as the sonde ascended for 45 minutes. Nonetheless, using dry days the minimum variation in water vapor proved the best comparison and most stringent test of the far-IR line wing absorption, a primary objective of the RHUBC-II campaign. 


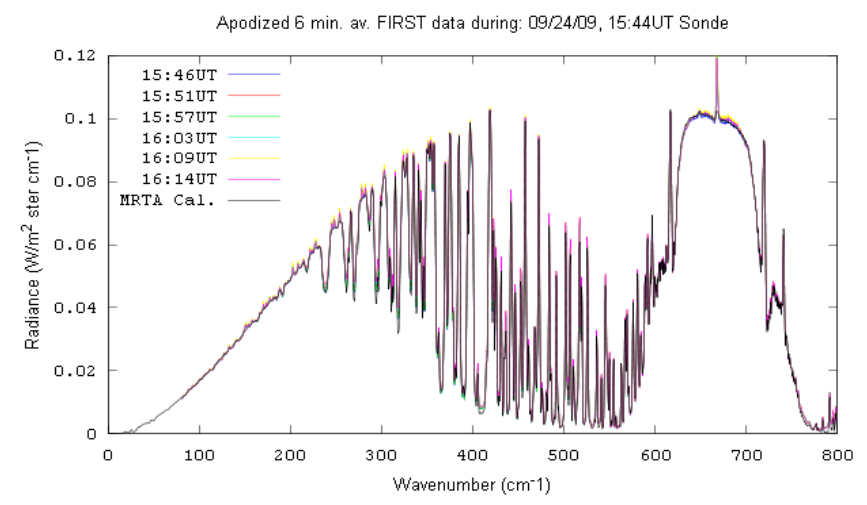

Figure 3

Line structure in the data, and produced by MRTA and other LBL models, below about $500 \mathrm{~cm}^{-1}$ is mainly due to the pure rotational structure of water vapor (see Figure 3). The band centered at $667 \mathrm{~cm}^{-1}$ is due to $\mathrm{CO}_{2}$. In the center of the intensely absorbing $\mathrm{CO}_{2}$ band, the brightness temperature is that of the near-to-surface atmosphere because above this height the atmosphere is opaque. In a window region, between about 800 and $1250 \mathrm{~cm}^{-1}$, the brightness temperature is that of the coldest part of the atmosphere above the site.

The absorption around the center of a spectral line of water vapor is very closely described by the Voigt line shape. The precise shape and properties of the absorption lines farther from line center are not well understood. Absorption in these "far wings" of the lines is stronger than is predicted by current collisional (pressure) or Doppler line broadening theories. Though the physics of this "missing" absorption are not well understood, an empirical model for the "continuum" absorption due to line wing overlap has been relatively successful in describing the available atmospheric infrared radiance data

The application of this formulation of the continuum absorption model modification to the strict line-byline code to the water vapor continuum (MT_CKD) ${ }^{18,19}$ has been tuned to observations at infrared wavelengths shorter than $17 \mu \mathrm{m}$ (less than $590 \mathrm{~cm}^{-1}$ ) with AERI data. ${ }^{20}$ Estimates of the downwelling longwave radiation at the surface increase by almost $6 \mathrm{~W} \mathrm{~m}^{-2} \mathrm{sr}^{-1}$, or $5.8 \%$, due to application of this continuum correction model. However, where data is not available, longward of $17 \mu \mathrm{m}$ (wavenumbers below $400 \mathrm{~cm}^{-1}$ ) this continuum absorption formulation for the radiative transfer remains untested. Data from FIRST from 100 to $600 \mathrm{~cm}^{-1}$ can be applied to this task.

\subsection{FIRST Data and MRTA Calculation Comparison}

Difference between the MRTA model calculations, with sonde data at times comparable to the observations, and the observed radiances with FIRST, is shown below in Figure 4. 


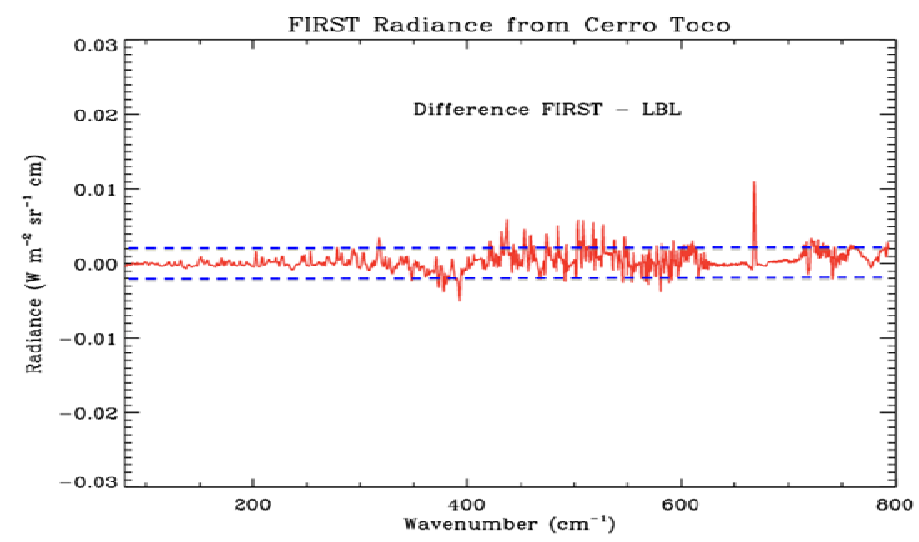

Figure 4

The model calculated spectral resolution is reduced to the FIRST data resolution and apodized with the same von Hann function. The results, peak-to-peak radiance difference of $6 \mathrm{~mW} /\left(\mathrm{m}^{2}\right.$ ster $\left.\mathrm{cm}^{-1}\right)[\sim 6 \%$ of a $270 \mathrm{~K}$ black body radiance], and average values, noted above, approximately $1 / 3$ of that value, are very similar in magnitude to the differences noted in both the REFIR and AERI data sets when compared to FIRST data.

To investigate the model differences further, and determine if there were any patterns or trends in these differences that might indicate continuum modeled absorption improvements, the FIRST data and MRTA model differences were first normalized with a $270 \mathrm{~K}$ black body function to remove the radiance variation due to this function. The resulting normalized differences were then plotted versus the normalized MRTA calculated radiance values to the same black body function; this to enhance the lower values of MRTA radiance indicative of line wing absorption. The data were then binned by wavenumber in $100 \mathrm{~cm}^{-1}$ bins to help show where in the spectrum the differences were most acute and if there was a trend was aligned with low absorption (low radiance) feature depth in the spectrum.
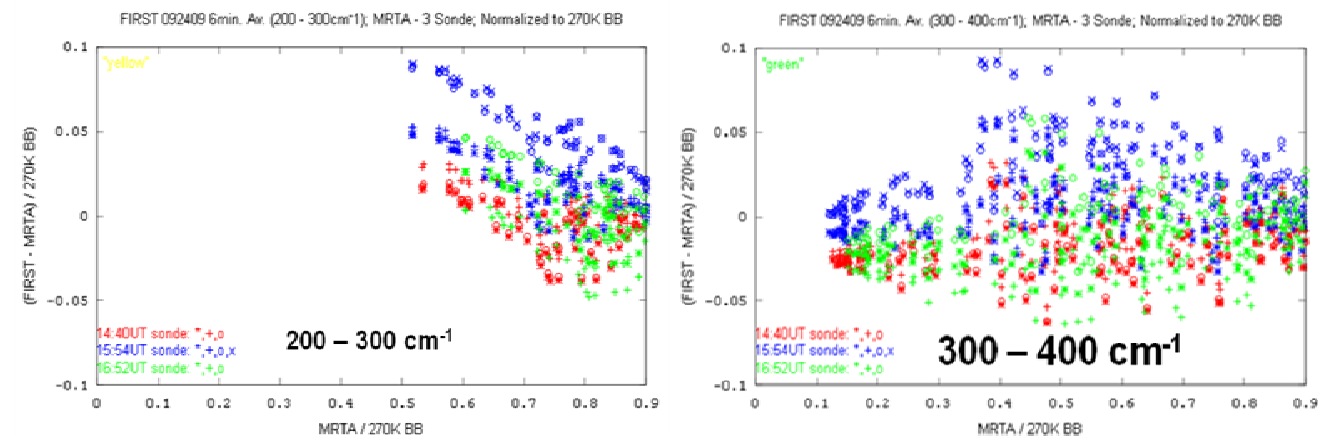

Figure 5a, 5b 

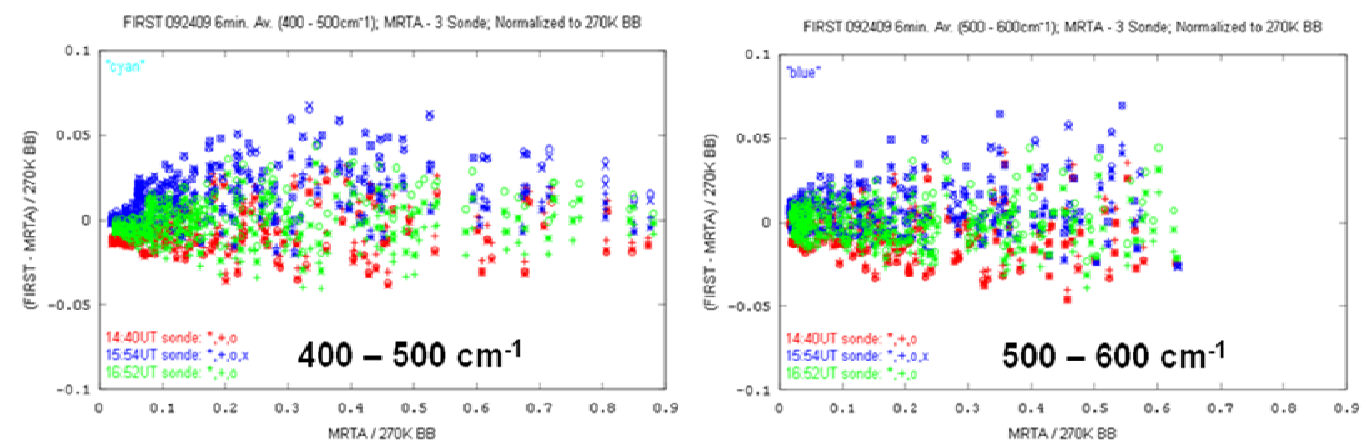

Figure 5c, 5d

The results of this normalizing and binning are shown in figures $5 \mathrm{a}$ through $5 \mathrm{~d}$ for wavenumber bins from 200 to $600 \mathrm{~cm}^{-1}$. These are plotted for 10 total FIRST 6 min. average data sets on Sept. 24, 2009, and associated by color (red, blue, and green) with three MRTA radiance calculations based on data sondes acquiring data at nearly the same time as the FIRST instrument. Differences between data points of the different colors are likely real changes in atmospheric water vapor during the flight of each sonde, though upon close inspection there is consistency of the differences in the model and data from one data set to another.

Overall, there is significant scatter in the data in Figure 5b, 5c, and 5d, not unexpected for a measurement occurring under conditions wherein real water vapor changes are occurring in a highly structured portion of the spectrum where line mixing and uncertainties in model absorption cross section can play a role. However, in Figure 5a, the 200-300 cm-1 bin, there is a definite trend in the differences between the data and the model which increases toward smaller values in normalized MRTA radiances. These occur in the wings of water vapor lines, where the absorption, and therefore radiance are lowest, implying an adjustment may be needed in the LBL code continuum model absorption to match the data and higher radiances in these line wings.

Calculations of the radiative transfer with the LBL code MRTA reported here applied MT_CKD 2.1 to the radiance calculation. An adjusted continuum model, MT_CKD 2.4, was recently added to MRTA code to apply a modified continuum absorption for water vapor. To test the assumption that figure 5a might imply continuum adjustment is required in the $200-300 \mathrm{~cm}^{-1}$ region, radiances were calculated with MRTA using version 2.4 with the same input parameters (absorbers, HITRAN line list version, water vapor profile, etc.). The differences between the modeled radiances with the two continuum models were in the region 200 and $500 \mathrm{~cm}^{-1}$ but were no larger than $1.2 \%$ (relative to a 270K black body) at approximately $300 \mathrm{~cm}^{-1}$. 


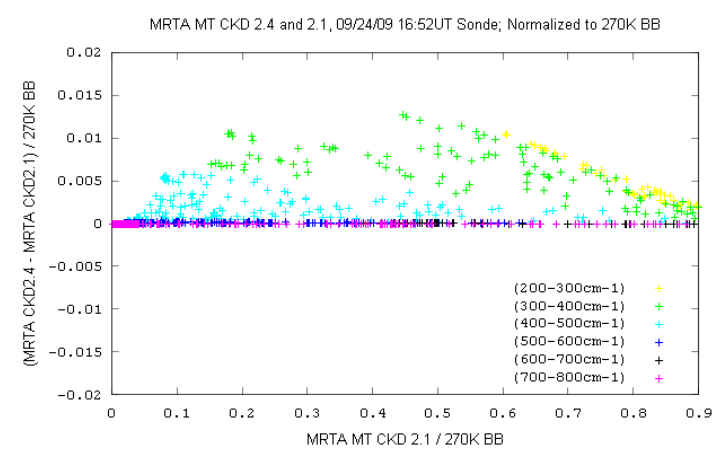

Figure 6

Version 2.4 and 2.1 continuum model calculations with MRTA were differenced and normalized to a 270K blackbody and divided into the same wavelength bins as the data sets in Figure 5 (see Figure 6). The shape and scatter in the continuum model comparisons is the same, though the magnitude of the differences in the continuum model is only $20 \%$ of the difference in the version 2.1 continuum model and data differences; perhaps implying that further adjustment (an update in version 2.4), particularly in the 200 to $300 \mathrm{~cm}^{-1}$ region, is required in the continuum model to match the FIRST data radiances.

\section{Conclusions}

There is reasonable agreement between in the preliminary instrument data sets from the Cerro Toco and models based on timed sondes when matching line shape and spectral resolution (here using the same $0.643 \mathrm{~cm}^{-1}$ resolution with von Hann apodization). At least during the most stable dry days, these radiance differences are typically within $2-3 \%$ and do not exceed 7\% PTP. Some of the PTP differences may be accounted for with improved instrument data correction techniques for instrument line shapes and wavenumber scale. When compared with a LBL model FIRST calibrated and normalized radiances are typically within $+/-5 \%$ of the modeled radiance (compared to the $270 \mathrm{~K}$ blackbody radiance) in the highly structured water vapor rotational bands and $+/-2 \%$ for the higher radiance $\mathrm{CO}_{2}$ band through to an atmospheric window region, $600-800 \mathrm{~cm}^{-1}$. The FIRST data set further implies that there may be the need for LBL continuum model adjustment, particularly in the $200-300 \mathrm{~cm}^{-1}$ region. In this paper, a method is outlined to test for this meeting a major objective of RHUBC-II campaign. Further analysis of the FIRST data set could also extend the AERI data overlap region with overlap from 1200 to $2000 \mathrm{~cm}^{-1}$, once polypropylene beamsplitter absorption corrections are applied.

\section{Acknowledgements}

The authors would like to thank the NASA Earth Science and Technology Office for FIRST Instrument Incubator Project support though balloon flight deployment, the Space Dynamics Laboratory (SDL) at Utah State University, Logan, UT for instrument operations support, the NASA Langley Research Center, for equipment support of the Far-infrared Observations of the Radiative Greenhouse Effect as a contribution to the RHUBC-II campaign, funding support from the NASA HQ Radiation Sciences Division, Cerro Toco logistics and field deployment on-site support from the Department of Energy's 
Atmospheric Radiation Monitoring group and the Atacama Large Millimeter Array, as well as the Atmospheric and Environmental Research, Inc. and University of Wisconsin, Madison, for analytical, sonde and AERI data reduction support.

\section{References}

1. M. G. Mlynczak, et al., "First light from the Far-Infrared Spectroscopy of the Troposphere (FIRST) instrument,” Geophys. Res. Lett. 33, L07704, doi:10.1029/2005GL025114, 2006.

2. R. O. Knuteson, et al., “Atmospheric Emitted Radiance Interferometer. Part I: Instrument design,” J. Atmos. Oceanic Technol. 21, pp. 1763-1776, 2004 a.

3. G. Bianchini, L. Palchetti, and B. Carli, "A wide-band nadir-sounding spectroradiometer for the characterization of the Earth's outgoing long-wave radiation,” Systems and Next-generations Satellites XII 6361, pp. U64-U74, SPIE, 2006.

4. S. Clough, P. Brown, N. Miller, J. Liljegren, and T. Shippert, Residual analysis of surface spectral radiances between instrument observations and line-by-line calculations, in Proceedings of the Fourth Atmospheric Radiation Measurement (ARM) Science Team Meeting, U.S. Department of Energy, 1994.

5. D. Kratz, "High-resolution modeling of the far infrared," in Optical and Spectroscopic Techniques, Remote Sensing, and Instrumentation for Atmospheric and Space Research IV, 4485, pp. 171-180, SPIE, 2001.

6. F. Miskolczi and M. G. Mlynczak, "The greenhouse effect and the spectral decomposition of the clear-sky terrestrial radiation,” Idojaras 108, pp. 209-251, 2004.

7. J. Harries, B. Carli, R. Rizzi, C. Serio, M. Mlynczak, L. Palchetti, T. Maestri, H. Brindley, and G. Masiello , “The far-infrared earth,” Rev. Geophys. 46, RG4004, doi:10.1029/2007RG000233, 2008.

8. B. A. Wielicki, et al., "Evidence for large decadal variability in the tropical mean radiative energy budget,” Science 295, pp. 841-844, 2002.

9. R. A. Hanel, B. Schlachman, F. D. Clark, C. H. Prokesh, J. B. Taylor, W. M. Wilson, and L. Chaney, “The Nimbus III Michelson interferometer,” Appl. Opt. 9, pp. 1767-1774, 1970.

10. R. A. Hanel, B. Schlachman, D. Rogers, and D. Vanous, "Nimbus 4 Michelson interferometer," Appl. Opt., 10, pp. 1376-1382, 1971.

11. D. Spankuch and W. Dohler, "Radiative properties of cirrus clouds in the middle IR derived from Fourier spectrometer measurements from space,” Z. Meteorol. 35, pp. 314-324, 1985.

12. H. E. Revercomb, H. Juijs, H. B. Howell, D. D. LaPorte, W. L. Smith, and L. A. Sromovsky, "Radiometric calibration of IR Fourier transform spectrometers: Solution to a problem with the High-Resolution Interferometer Sounder,” Appl. Opt. 27, pp. 3210-3218, 1988. 
13. R. O. Knuteson, et al., “Atmospheric Emitted Radiance Interferometer. Part II: Instrument performance,” J. Atmos. Oceanic Technol. 21, pp. 1777-1789, $2004 \mathrm{~b}$.

14. A. G. Bianchini, and L. Palchetti, "Technical Note: REFIR-PAD level 1 data analysis and performance characterization,” Atmos. Chem. Phys. 8, pp. 3817-3826, 2008.

15. L. Miloshevich, H. Vomel, D. Whiteman, and T. Leblanc, "Accuracy assessment and correction of Vaisala RS92 Radiosonde water vapor measurements,” J. Geophys. Res., B, doi:10.1029/2008JD011565, 2009.

16. D. P. Kratz, et al., “An inter-comparison of far-infrared line-by-line radiative transfer models,” $J$. Quant. Spectrosc. Radiat. Transfer 90, pp. 323-341, 2005.

17. L. S. Rothman, et al., "The HITRAN 2008 molecular spectroscopic database,” J. Quant. Spectrosc. Radiat. Transfer 96, pp. 139-204, 2009.

18. S. A. Clough, F. X. Kneizys, and R. W. Davies, "Line shape and the water vapor continuum,” Atmos. Res. 23, pp. 229-241, 1989.

19. E. J. Mlawer, S. A. Clough, P. D. Brown, and D. C. Tobin, "Recent developments in the water vapor continuum,” Ninth ARM Science Team Meeting, U. S. Dep. Of Energy, San Antonio, Tex., 22-26 March, 1999.

20. D. C. Tobin, et al., "Downwelling spectral radiance observations at the SHEBA ice station: Water vapor continuum measurements from 17 to 6 mm,” J. Geophys. Res., 104, pp. 2081-2092, 1999. 